\title{
Possible Mechanisms of Single and Multiple Star Formation
}

\author{
G. J. Odgers, Dominion Astrophysical Observatory, Victoria, B. C., Canada
}

AND

\author{
R. W. Stewart, Department of Physics, University of British Columbia, Vancouver, B. C., Canada
}

$I^{\mathrm{F}}$ F we suppose that at least some stars are the result of the gravitational collapse of $\mathrm{HI}$ clouds, it seems necessary to consider Jeans' criterion. This can be derived by a number of arguments, but for our purpose it is sufficient to consider it a result of dimensional analysis, and we get

$$
V^{2} \leq G M / L,
$$

where $G$ is the gravitational constant, $M$ is a typical dimension, and $V$ is a characteristic velocity comprising all velocities interior to the region of gas being considered.

The criterion remains unaffected by the presence of a magnetic field because a motion in which the density waves are perpendicular to the lines of force has the particle motion parallel to the lines of force and is unaffected by the field. Further, it can be shown ${ }^{1}$ that a density wave forming an angle with the lines of force can be obtained by particle motions parallel to these lines. The details of the actual motion, however, depend critically on the nature of the magnetic field.

If we apply this criterion to what is known of $\mathrm{HI}$ clouds, we find that a rather large cloud with $L$ of the order of $100 \mathrm{pc}$ and $M$ about $10^{5}-10^{6}$ solar masses is required for instability. There seems to be no reason why we should not accept this estimate, so we use it as a starting point. Actually, a rather denser than average region on which there is imposed some initial centripetal motion, perhaps due to collisions, may be required to overcome time-scale difficulties.

Once the gas cloud becomes unstable it will begin to collapse. The collapse is resisted by the internal stresses which are due to thermal motions, to turbulent and shock wave motions, and to the magnetic field. These stresses gain energy at the expense of gravitational potential energy, but if the loss of energy to radiation is sufficient they are unable to prevent collapse.

The rate of turbulent energy density decay is proportional to $\rho V^{3} / L$. We had $V^{2} \sim G M / L$, and note that $\rho \sim L^{-3}$, so that the rate of energy dissipation $\sim L^{-11 / 2}$. However, the rate of radiation of energy will most probably be proportional to the collision rate, i.e., to $\rho^{2}$ or to $L^{-6}$, so that the radiation of energy can at least keep pace with the dissipation.

Eventually, we obtain self-stable protostars with large $(20 \mathrm{~km} / \mathrm{sec})$ random velocities. If these stars are

\footnotetext{
${ }^{1}$ S. Chandrasekhar and E. Fermi, Astrophys. J. 118, 116 (1953).
}

sufficiently hot, the cloud can be blown apart, leaving an expanding association.

In order to obtain some notion of the role turbulence plays in star formation, consider a mass of gas contracting in its own plane of rotation and assume a linear change of size by a factor $q$. Conservation of angular momentum then requires that the angular velocities change by factors $q^{-2}$ and linear velocities by $q^{-1}$. Further, the internal motions become intensified by the contraction even if these motions are irrotational, ${ }^{2}$ for the following reason. The internal motions give rise to Reynolds' stresses on the gas which may or may not have shear-type components $\left\langle u_{i} u_{j}\right\rangle(i \neq j)$, but which always have normal components $\left\langle u_{i}{ }^{2}\right\rangle$ and during contraction work must be done against these stresses. This work appears as increased kinetic energy, so that the contraction increases the intensity of the internal movements; with the density increase this augments the amplitude of the pressure fluctuations.

It seems likely then that the gravitational potential energy is transformed into mass motions which carry most of the stress. But, unlike the pressure, the total stress is not then independent of the size of the portion of fluid considered so that a part of the original mass is acted on by stresses due only to motions internal to itself.

Since in the typical spectrum of turbulence most of the energy and stress is carried by the larger scales of motion, these internal stresses must be small if the contraction under gravity of this much smaller mass is to be possible.

It is interesting to note that one can show a mode of instability of the original contracting mass without considering turbulence at all. Jeans' criterion can be written

$$
L^{2} \geq V^{2} / G \rho \text {. }
$$

Suppose that a contraction by a factor of $q$ takes place in the linear dimensions; then, if the mass subdivides into $n$ equal parts, let $L^{*}, \rho^{*}$ be the size and density of one of the subdivisions so that $\rho^{*}=\rho q^{-3}, L^{*}=q n^{-\frac{1}{3}} L$; then if $V$ remains unaltered

$$
\text { if } \begin{aligned}
L^{* 2} & >V^{2} / G \rho^{*} \\
q^{-1} & \geq n^{3}
\end{aligned}
$$

${ }^{2}$ G. J. Odgers and R. W. Stewart, J. Roy. Astron. Soc. Canada 51, 97 (1957). 


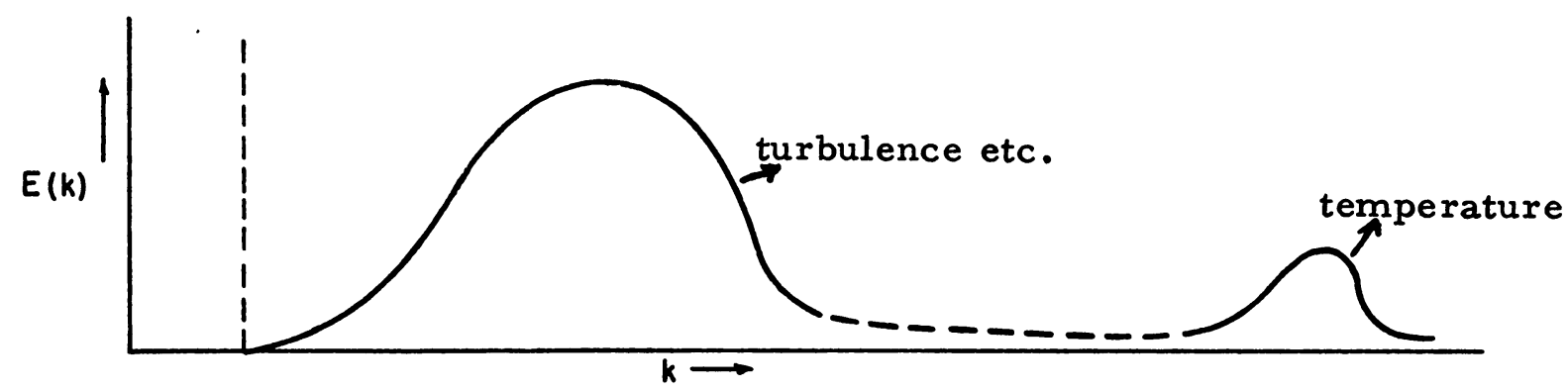

Fig. 1.

i.e., new subdivisions are gravitationally unstable if their number does not exceed $q^{-\frac{3}{2}}{ }^{2}$

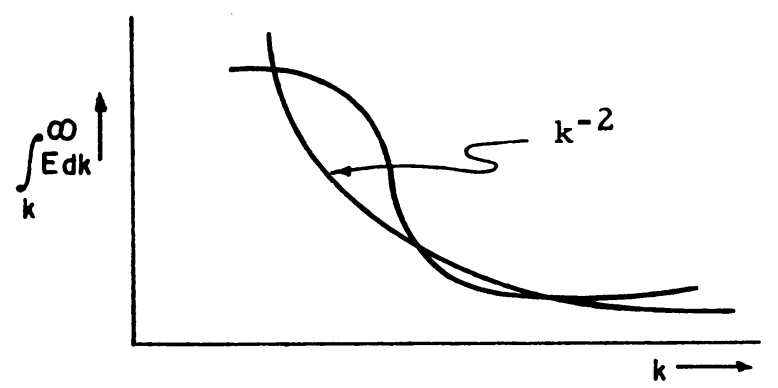

Fig. 2.

These two viewpoints can probably be combined if we consider these subdivisions as the large eddies in the turbulent fluid.
Consider now the hypothetical spectrum of turbulence shown in Fig. 1. For any volume of dimensions $k^{-1}$ the internal stress is determined by $\int_{k}^{\infty} E(k) d k$, see Fig. 2. The critical size varies as $M^{3}$, that is as $k^{-2}$. If the $k^{-2}$ curve cuts the $\int_{k}^{0} E(k) d k$ curve at any point, we shall have there an instability and subdivision with collapse into much smaller masses. Note that such a subdivision will naturally occur into very small fractions of the original, because from the nature of the turbulent spectrum we cannot expect our two curves to intersect until quite large values of $k$ relative to that value characteristic of the original gas cloud. If we consider the spectrum to drop off as $k^{-5 / 3}$ no cut can occur, but this range does not continue long-if it exists at all-and is followed by a much steeper drop. It is required, however, that the instability be reached before temperature effects become important. That this is likely has been shown in the foregoing.

\section{DISCUSSION}

D. L. LAYZER, Harvard College Observatory, Cambridge, Massachusetts: I should like to question whether permanent condensations can form in a cloud contracting under its own gravitation. While I am not familiar with the details of Stewart's calculations, I recall that similar calculations seem to show that this kind of instability does not occur. A small element in a selfgravitating cloud is usually not cool enough to be stable under its own gravitation. The formula that Stewart has written $v^{2} \sim G M / L$, or $v^{2} \sim G \rho L^{2}$-shows that in a cloud that is in equilibrium, or slowly contracting, the kinetic energy of a small condensation is too great by a factor not much smaller than $(L / l)^{2}-l$ being a dimension of the condensation-for the condensation to be in equilibrium under its own gravitation. Now, I think Stewart would like to reduce the kinetic energy of the condensation by putting some of it into motions on a larger scale. This will help. But if one uses Kolmogoroff's law, he finds that the kinetic energy of small condensations is still too great, by a large factor, for them to be self-gravitating. I think the situation is even more unfavorable when one takes compressibility into account.
R. W. STEwarT, Department of Physics, University of British Columbia, Vancouver, B. C., Canada: Of course, this would give me a beautiful opportunity to make rude remarks about the Kolmogoroff law, but I will not do that. The main thing that I did not state here through an oversight was that what is demanded is an enormous increase in density. I am looking here for a collapse where the cloud which was originally at about 100 pc is down to about $1 \mathrm{pc}$ in diameter, and the density is then of the order of $10^{6}$ atoms per cubic centimeter. Now, you can in fact put some numbers in and show that if the temperature will stay down, that this will now subdivide to that extent.

D. L. LAYZER : Putting numbers into these formulas may obscure what is going on. It is true that if the temperature is sufficiently low, Jeans' criterion will predict that small elements are unstable and must contract. But under these conditions the cloud as a whole must contract at essentially the same rateindeed faster. So the condensations will not separate out. 
R. W. STEWART: I think we had better discuss this privately. It is just a matter of relative rates which we have actually computed.

G. K. BATChELOR, Trinity College, Cambridge England: There is a small piece of work described in the proceedings of the last Symposium which might be recalled at this point. I do not think it changes what Stewart said, but it is related to it. It was shown in those proceedings that one could derive the effect of a rapid contraction on the energy of the turbulence in the medium and, speaking from memory, the result comes out quite simply as being the same as if the medium were a perfect gas with just 3 degrees of freedom. The turbulent energy in unit volume can be thought of as being equivalent to a pressure which is additional to the gas pressure, and which will vary as the volume of the whole cloud raised to the power $-\gamma$, where $\gamma$ turns out to be $5 / 3$.

R. W. STEWART: Of course. If $\gamma$ is $5 / 3$, the thing will not collapse at all. It has to be better than $4 / 3$, but the point is that if one uses $5 / 3$, one has not taken account of the decay of the turbulent energy. One has to invoke that to get the whole picture. 